ARTICLE

\title{
Cryptochrome-mediated blue-light signalling modulates UVR8 photoreceptor activity and contributes to UV-B tolerance in Arabidopsis
}

\author{
Nicolas Tissot (iD) ${ }^{1,2} \&$ Roman Ulm (i) ${ }^{1,2 凶}$
}

UV-B constitutes a critical part of the sunlight reaching the earth surface. The homodimeric plant UV-B photoreceptor UV RESISTANCE LOCUS 8 (UVR8) monomerizes in response to $U V-B$ and induces photomorphogenic responses, including UV-B acclimation and tolerance. REPRESSOR OF UV-B PHOTOMORPHOGENESIS 1 (RUP1) and RUP2 are negative feedback regulators that operate by facilitating UVR8 ground state reversion through re-dimerization. Here we show that RUP1 and RUP2 are transcriptionally induced by cryptochrome photoreceptors in response to blue light, which is dependent on the bZIP transcriptional regulator ELONGATED HYPOCOTYL 5 (HY5). Elevated RUP1 and RUP2 levels under blue light enhance UVR8 re-dimerization, thereby negatively regulating UVR8 signalling and providing photoreceptor pathway cross-regulation in a polychromatic light environment, as is the case in nature. We further show that cryptochrome 1 , as well as the red-light photoreceptor phytochrome B, contribute to UV-B tolerance redundantly with UVR8. Thus, photoreceptors for both visible light and UV-B regulate UV-B tolerance through an intricate interplay allowing the integration of diverse sunlight signals.

\footnotetext{
${ }^{1}$ Department of Botany and Plant Biology, Section of Biology, Faculty of Sciences, University of Geneva, 30 Quai E. Ansermet, 1211 Geneva 4, Switzerland.

${ }^{2}$ Institute of Genetics and Genomics of Geneva (iGE3), University of Geneva, Geneva, Switzerland. ${ }^{凶}$ email: roman.ulm@unige.ch
} 
S unlight is an essential factor for life on earth. It is the ultimate source of energy for photosynthesis and it modulates plant growth and development. Flowering plants can perceive light signals spanning from UV-B to far-red $(\sim 280-800$ $\mathrm{nm})$ using five classes of photoreceptors ${ }^{1}$. UV-B $(280-315 \mathrm{~nm})$ induces photomorphogenic responses and UV-B acclimation ${ }^{2-5}$. The UV-B photoreceptor UV RESISTANCE LOCUS 8 (UVR8) forms homodimers in its ground state and monomerizes to the active form in response to UV-B absorption ${ }^{6,7}$. UVR8 monomers interact directly with the WD40 domain of the E3 ubiquitin ligase CONSTITUTIVELY PHOTOMORPHOGENIC 1 (COP1 $)^{5,7}$. The UVR8-COP1 interaction competes with that between COP1 and the photomorphogenesis-promoting bZIP transcription factor ELONGATED HYPOCOTYL 5 (HY5), thereby preventing its ubiquitination $^{8,9}$. This leads to stabilization of HY5 and the induction of many target genes encoding proteins involved in the photomorphogenic response to UV-B, including HY5 itself ${ }^{10-12}$. Among these HY5-induced genes, REPRESSOR OF UV-B PHOTOMORPHOGENESIS 1 (RUP1) and RUP2 encode WD40-repeat proteins that provide UVR8 negative feedback regulation ${ }^{13}$. RUP1 and RUP2 directly interact with UVR8 to facilitate its redimerization, thereby inactivating the UVR8 monomer ${ }^{14,15}$. RUP1 and RUP2 can also be part of a CUL4-DDB1-based E3 ubiquitin ligase that targets HY5 for degradation ${ }^{16}$. Moreover, it has been proposed that COP1 directly targets RUP1 and RUP2 for ubiquitination and degradation under UV-B, contributing to the stabilization of HY5 $5^{16}$.

Cryptochrome blue-light signalling shows some interesting similarities to UVR8 UV-B signalling. The oligomeric state of cryptochromes changes in response to blue-light perception, specifically from an inactive monomeric to an active homodimeric state ${ }^{17}$. BLUE-LIGHT INHIBITOR OF CRYPTOCHROMES (BIC1) and BIC2 provide negative feedback regulation by directly binding to cryptochromes and inhibiting their dimerization ${ }^{17,18}$. Finally, active cryptochromes also inhibit the COP1 E3 ubiquitin ligase complex, which results in HY5 stabilization and accumulation ${ }^{9,19-24}$. Moreover, synergisms and interplays between cryptochrome and UV-B/UVR8 signalling have been described before; however, these remain poorly understood at the molecular level ${ }^{25-28}$.

Here, we show that induction of RUP1 and RUP2 gene expression and their ensuing protein accumulation are blue-light responsive. These inductions depend mainly on the blue-light photoreceptor cryl, through the activity of HY5, with lesser roles played by cry 2 and phyA. Enhanced RUP1 and RUP2 levels under blue light affect the balance between UVR8 monomer and UVR8 homodimer, thereby modulating the activity of the UV-B signalling pathway. Finally, we demonstrate that cryl, phyB, and UVR8 redundantly regulate UV-B tolerance.

\section{Results}

Cryptochromes and phyA activate RUP1 and RUP2 expression. Blue-light exposure of Arabidopsis seedlings resulted in strong and transient induction of RUP1 and RUP2 expression in wild type, but not in hy5 (Fig. 1a, b). In agreement, RUP2 protein accumulated in response to blue light in wild type, but not in hy5 to a detectable level (Fig. 1c). To identify the photoreceptors responsible for the blue-light induction of RUP1 and RUP2 expression, we examined responses in $\operatorname{cry} 1, \operatorname{cr} y 2, \operatorname{cry} 1$ cry 2 , and cry 1 cry 2 phyA. cry 1 and cry 2 single mutants both displayed reduced blue-light induction of RUP1 and RUP2, which was further reduced in cry 1 cry 2 double mutants and absent in cry 1 cry 2 phyA triple mutants (Fig. 1d, e). In agreement, RUP2 protein accumulation in response to blue light was reduced in cry1, cry2, and $p h y A$, further reduced in $c r y 1$ cry 2 , and undetectable in cry 1 cry2 phyA (Fig. 1f). The absence of an anti-RUP1 antibody prevented directly testing endogenous RUP1 levels. We conclude that blue-light-dependent cryptochrome and phyA signalling activates RUP1 and RUP2 expression, resulting in RUP2, and likely RUP1, protein accumulation.

cry1 and phyA signalling enhances UVR8 re-dimerization. Accumulation of RUP1 and RUP2 in response to blue light points to a previously unknown effect of blue-light signalling on UVR8 activity. We thus tested the effect of blue light on the dynamics of the UVR8 homodimer/monomer ratio upon UV-B treatment, with a particular focus on UVR8 re-dimerization post UV-B exposure (Fig. 2a). The UV-B treatment induced a strong UVR8 monomerization in wild type and rup1 rup2 but did not affect the total amount of UVR8 (-UV and +UV; Fig. 2b, c). During the subsequent recovery in darkness, UVR8 re-dimerization was significantly faster in wild-type seedlings that were pre-exposed to blue light than that in seedlings without blue-light treatment $\left(30^{\prime}\right.$ and $60^{\prime}$; Fig. 2b, c). This blue-light enhancement of UVR8 redimerization was absent in rup1 rup2 mutants, in which UVR8 remained monomeric during the 1 -h post-UV-B recovery (Fig. 2b, c). UVR8 re-dimerization was also impaired in rup1 and rup2 single mutants but to a considerably lesser extent than in the rup1 rup2 double mutant, supporting redundant roles of RUP1 and RUP2 in this process (Supplementary Fig. 1) ${ }^{13,14}$. Moreover, in agreement with the role of HY5 in RUP1 and RUP2 activation, blue-light-enhanced UVR8 re-dimerization was strongly impaired in hy5 mutants (Supplementary Fig. 2).

To test the roles of specific photoreceptors in blue-lightenhanced UVR8 re-dimerization, we quantified the UVR8dimer/ $\mathrm{UVR}^{\text {total }}$ ratio in wild-type seedlings and cry1, cry2, cry1 cry2, phyA, and cry1 cry 2 phyA mutant seedlings. Without blue-light pre-treatment, no differences were observed in UVR8 redimerization kinetics among the six tested genotypes (Fig. 2d, f). However, in blue-light pre-treated seedlings, the enhanced UVR8 re-dimerization apparent in wild type was partially impaired in phyA and considerably more so in cry 1 cry 2 and cry1 cry 2 phyA (Fig. 2e). This result agrees with the reduced RUP2 accumulation in response to blue light observed in these photoreceptor mutant lines (Fig. 1e). In further agreement with a key role of cry1, overexpression of GFP-cry1 resulted in elevated levels of RUP2 in response to blue light and further enhanced UVR8 re-dimerization when compared to wild type under these conditions (Supplementary Fig. 3). In contrast to cry 1 and cry 1 cry2, blue-light pre-treated cry2 single mutants displayed UVR8 re-dimerization kinetics comparable to that in wild type (Fig. $2 \mathrm{~g}$ ). We conclude that blue light enhances UVR8 re-dimerization, and that this is mainly due to cryl and phyA signalling-mediated RUP1 and RUP2 accumulation.

BIC overexpression blocks enhanced UVR8 re-dimerization. BIC1 and BIC2 are negative feedback regulators of cry1 and cry $2^{17}$. We therefore tested whether BIC1 and BIC2 accumulation could affect RUP1/RUP2 expression and RUP2 protein levels. Blue-light-induced accumulation of RUP1/RUP2 mRNA and RUP2 protein was indeed reduced in the BIC1 and BIC2 overexpression lines bic1-D1, BIC1-GFP, and BIC2-GFP to a similar level as in $c r y 1$ cry2 (Fig. 3a, b). This agrees with inhibitory roles of BIC1 and BIC2 in cry1 and cry 2 activities ${ }^{17}$. However, we did not observe enhanced RUP2 protein levels in the bic1 bic2 double mutant compared to that in wild type (Fig. $3 \mathrm{~b}$ ). To test whether BIC1 and BIC2 overexpression modulates UVR8 activity, we UV-B treated various genotypes that were pre-treated with blue light or not, and then quantified UVR8 re-dimerization after 30-min recovery. In agreement with the observed RUP2 levels, 

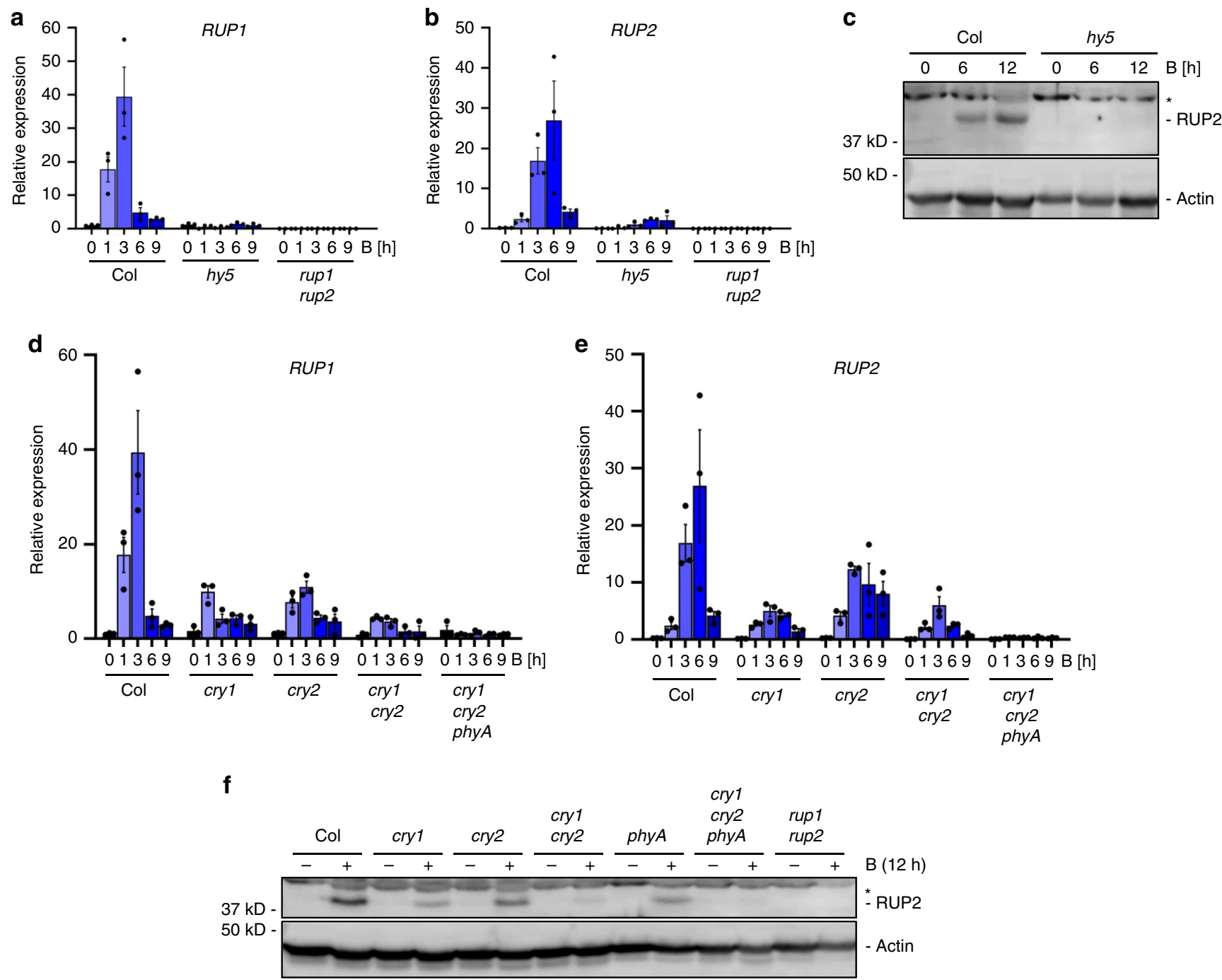

Fig. 1 Blue-light-induced RUP1 and RUP2 expression and RUP2 protein accumulation depend on cry1, cry2, phyA, and HY5. a, b qRT-PCR analysis of $\mathbf{a}$ RUP1 and $\mathbf{b}$ RUP2 expression in 4-d-old wild type (Col), hy5-215 (hy5), and rup1-1 rup2-1 (rup1 rup2) seedlings grown in darkness or treated with blue light $\left(\mathrm{B} ; 50 \mu \mathrm{mol} \mathrm{m} \mathrm{m}^{-2} \mathrm{~s}^{-1}\right)$ for $1,3,6$, or $9 \mathrm{~h}$. Data are means \pm SEM $(N=3)$. c Immunoblot analysis of RUP2 protein level in 4-d-old Col and hy5 seedlings grown in darkness $(0)$ or treated with blue light for 6 or $12 \mathrm{~h}$. The asterisk indicates a nonspecific cross-reacting band. Actin is shown as protein loading control. d, e qRT-PCR analysis of $\mathbf{d}$ RUP1 and e RUP2 expression in 4-d-old Col, cry1-304 (cry1), cry2-1 (cry2), cry1-304 cry2-1 (cry1 cry2), and cry1-304 cry2-1 phyA-412 (cry1 cry2 phy A) seedlings grown in darkness or treated with blue light for 1, 3, 6, or $9 \mathrm{~h}$. Data are means \pm SEM $(\mathrm{N}=3$ ). Note that the Col samples in panels (d) and (e) are identical to those in panels (a) and (b), respectively, as the data are derived from the same experiment. $\mathbf{f}$ Immunoblot analysis of RUP2 protein level in 4-d-old Col, cry1, cry2, cry1 cry2, phyA-211 (phyA), cry1 cry2 phyA, and rup1 rup2 seedlings grown in darkness, then treated with blue light for $12 \mathrm{~h}(+)$ or not $(-)$. The asterisk indicates a nonspecific cross-reacting band. Actin is shown as protein loading control.

blue-light-enhanced UVR8 re-dimerization was similar in bic1 bic2 and wild type, but strongly impaired in the BIC1 and BIC2 overexpression lines, comparable to that in cry 1 cry 2 and rup 1 rup2 (Fig. 3c). Interestingly, reciprocally to RUP1 and RUP2 activation through cryptochrome-mediated blue-light signalling (Fig. 1) $)^{13}$, BIC1 and BIC2 are induced by UVR8-mediated UV-B signalling (Fig. $3 \mathrm{~d})^{5}$. We conclude that inhibition of cryptochrome activity by $\mathrm{BIC} 1$ and $\mathrm{BIC} 2$ suppresses the blue-light enhancement of UVR8 re-dimerization, and that UVR8-mediated regulation of $B I C 1$ and $B I C 2$ may add an additional regulatory control level.

cry1 and cry2 repress UVR8-induced responses. The impact of blue-light signalling on UVR8 activity indicates an associated impact on UV-B responses. In comparison to wild type, cry1 cry 2 double mutants showed enhanced hypocotyl growth inhibition under supplementary UV-B (Supplementary Fig. 4a). It is of note, however, that cry1 cry 2 shows an elongated hypocotyl in the absence of UV-B compared to wild type, and a similar hypocotyl length in the presence of UV-B (Supplementary Fig. 4a). We thus reduced the UV-B to levels that were insufficient to induce hypocotyl growth inhibition in wild type to further test for UV-B hypersensitivity in cry 1 cry2. Strikingly, cry 1 cry 2 displayed a clear hypocotyl growth inhibition response under these conditions (Fig. 4a). This was further confirmed to be the case for a cryl mutant in the Ws accession (Supplementary Fig. 4b). A minor difference was also observed in UV-B-responsive anthocyanin accumulation between wild type and cry1 cry 2 under these conditions (Fig. 4b). We further investigated expression of UV-Bresponsive genes. We first compared the induction of $H Y 5$ and CHALCONE SYNTHASE (CHS) in cry1 cry2, uvr8, and wild type in response to 3 -h supplemental narrowband UV-B ${ }^{5,29}$. Whereas 
a

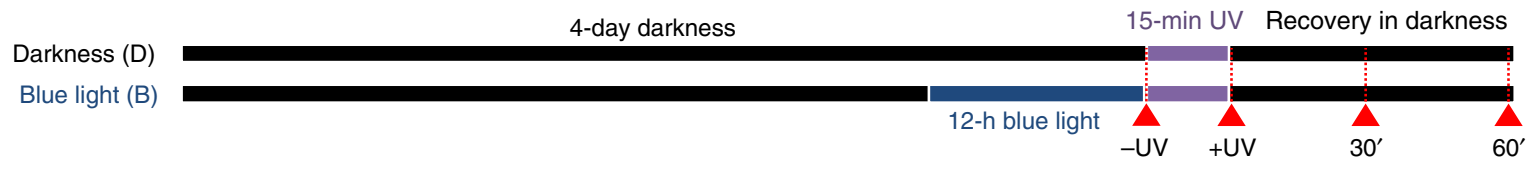

b $\frac{\text { Darkness }}{-U V+U V \quad 30^{\prime} \quad 60^{\prime}} \frac{\text { Blue light }}{-U V+U V ~ 30^{\prime} 60^{\prime}}$
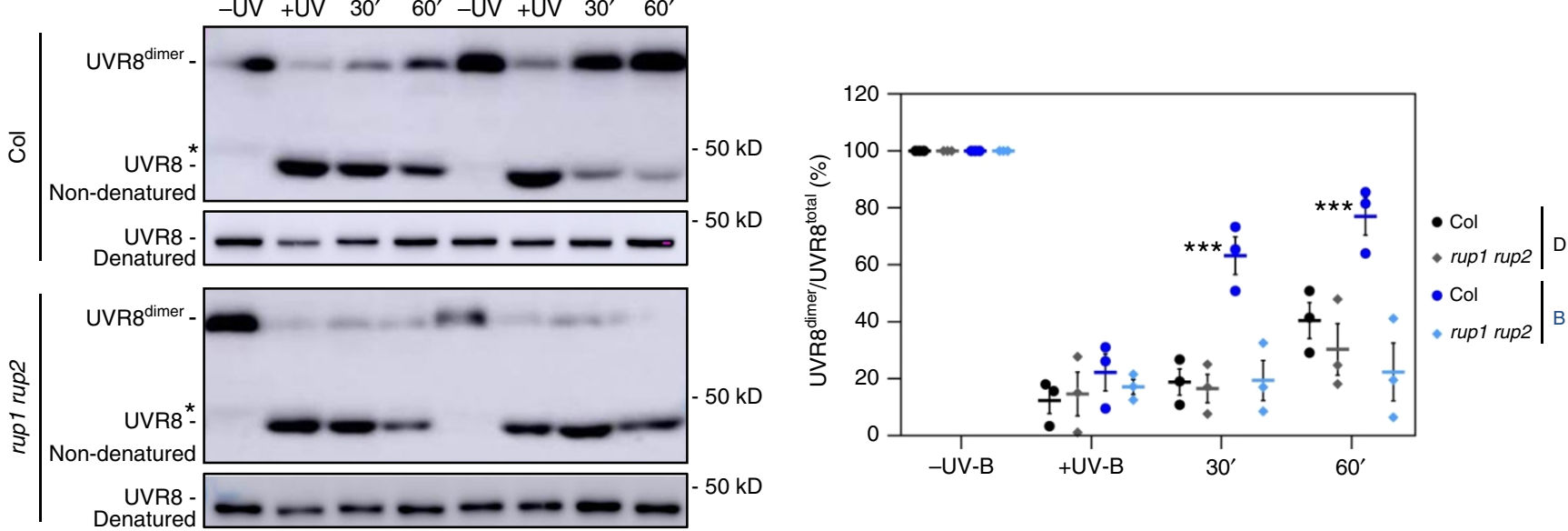

d

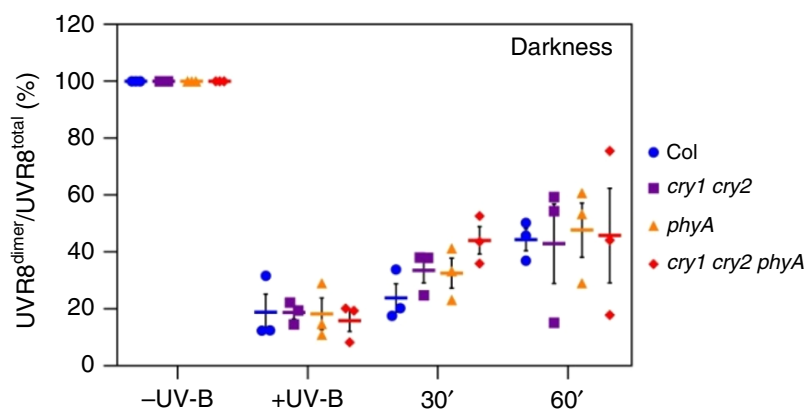

e

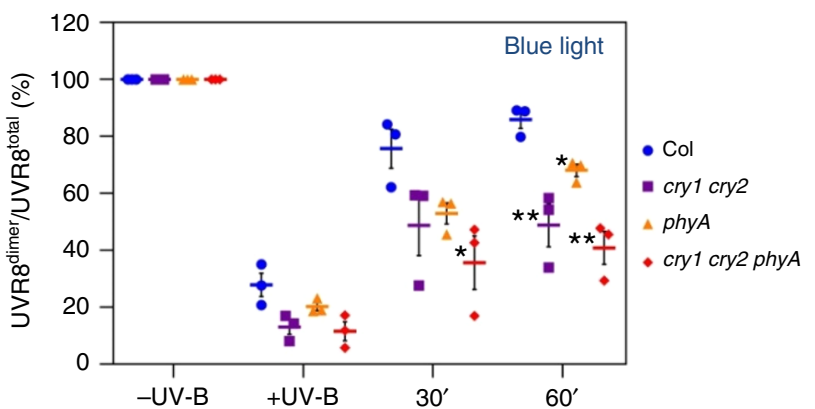

f

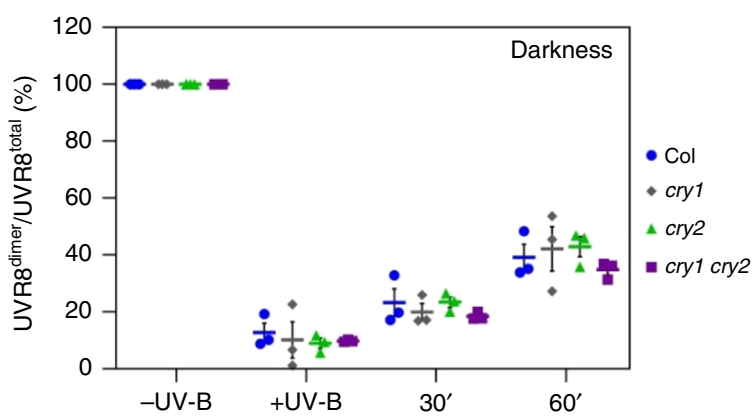

g

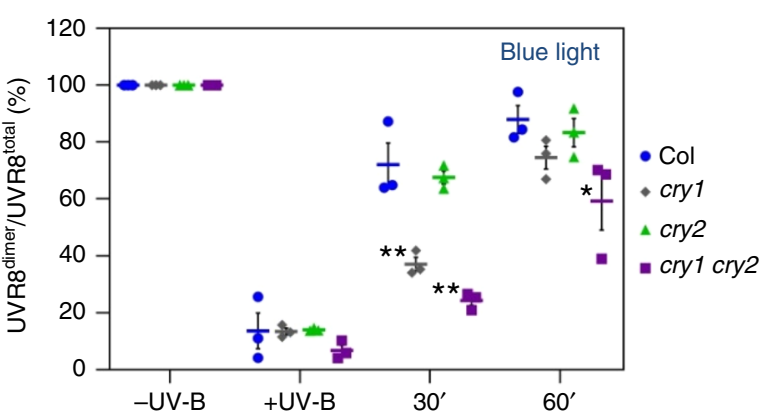

Fig. 2 cry1 and phyA are the main photoreceptors mediating the RUP1- and RUP2-dependent blue-light repression of UVR8 activity. a Schematic representation of the light treatments of seedlings for the UVR8 re-dimerization analyses. Four-d-old etiolated seedlings were exposed to 12-h blue light $\left(B, 50 \mu \mathrm{mol} \mathrm{m} \mathrm{m}^{-2} \mathrm{~s}^{-1}\right)$ or maintained in darkness $(D)$ before they were irradiated for 15 min with $U V-B\left(0.85 \mathrm{~mW} \mathrm{~cm}^{-2}\right)$ and maintained further in darkness for $1 \mathrm{~h}$. The time points of sampling are indicated by red arrows labelled with $-U V-B,+U V-B, 30^{\prime}$, and $60^{\prime}$. $\mathbf{b}$ Immunoblot analysis of UVR8 re-dimerization in wild type (Col) and rup1-1 rup2-1 (rup1 rup2) seedlings. UVR8 dimers were detectable in nonheat-denatured protein samples. Parallel denatured samples demonstrate equal amounts of UVR8 protein. The asterisk indicates a nonspecific cross-reacting band. c Quantification of the UVR8dimer/UVR8 ${ }^{\text {total }}$ ratio (\%) in Col and rup1 rup2. d, e Quantification of the UVR8dimer/UVR8total ratio (\%) in response to UV-B in Col, cry1-304 cry2-1 (cry1 cry2), phyA-211 (phyA), and cry1-304 cry2-1 phyA-412 (cry1 cry2 phyA) seedlings pre-treated with blue light (e) or not (d). f, $\mathbf{g}$ Quantification of the UVR8dimer/UVR8 ${ }^{\text {total }}$ ratio (\%) in response to UV-B in Col, cry1-304 (cry1), cry2-1 (cry2), and cry1 cry2 seedlings pre-treated with blue light (g) or not (f). Data are means \pm SEM ( $N=3$ ). ${ }^{\star} P<0.05,{ }^{\star \star} P<0.01$, and ${ }^{* \star *} P<0.001$.

uvr8 mutants did not respond to UV-B, the cry1 cry2 double mutant showed an enhanced activation of HY5 (7.8-fold) and CHS (329.5-fold) expression in response to UV-B in comparison to that in wild type (2.2- and 21.2-fold, respectively) (Fig. 4c, d, respectively). We further analysed the effect of cryl and cry 2 on
UVR8-mediated marker gene expression in response to treatment with 15-min broadband UV-B at three different irradiation levels followed by $45 \mathrm{~min}$ in white light (Fig. 4e). These UV-B irradiation conditions have previously been shown to induce expression of HY5, CHS, EARLY LIGHT-INDUCIBLE PROTEIN 
a
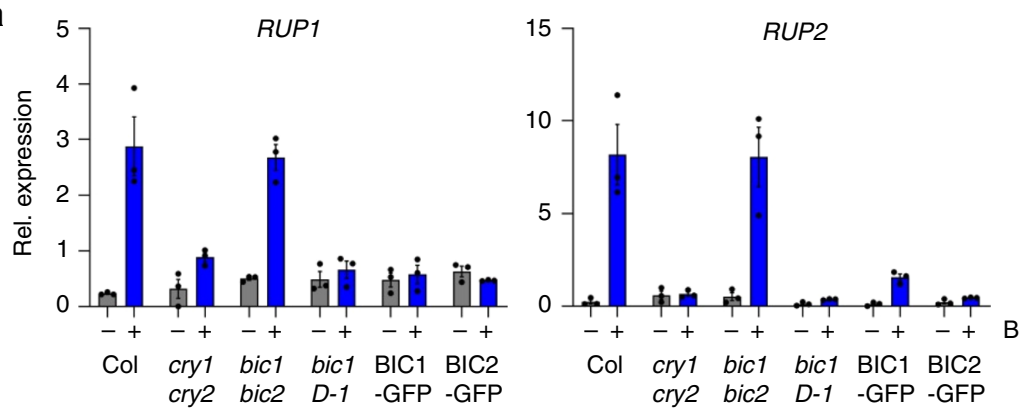

b

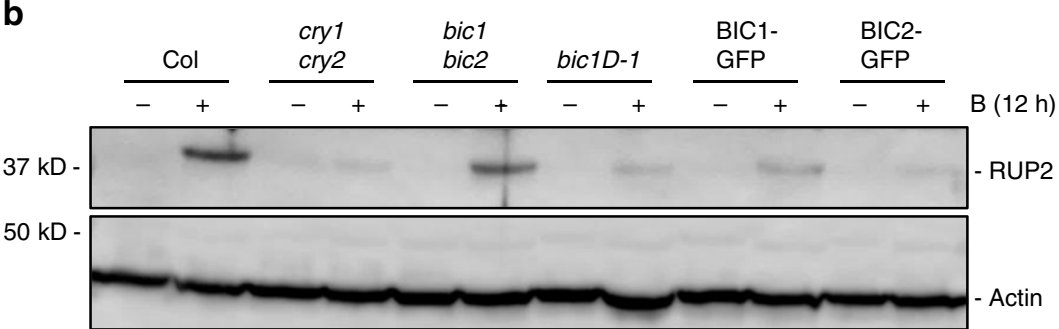

C

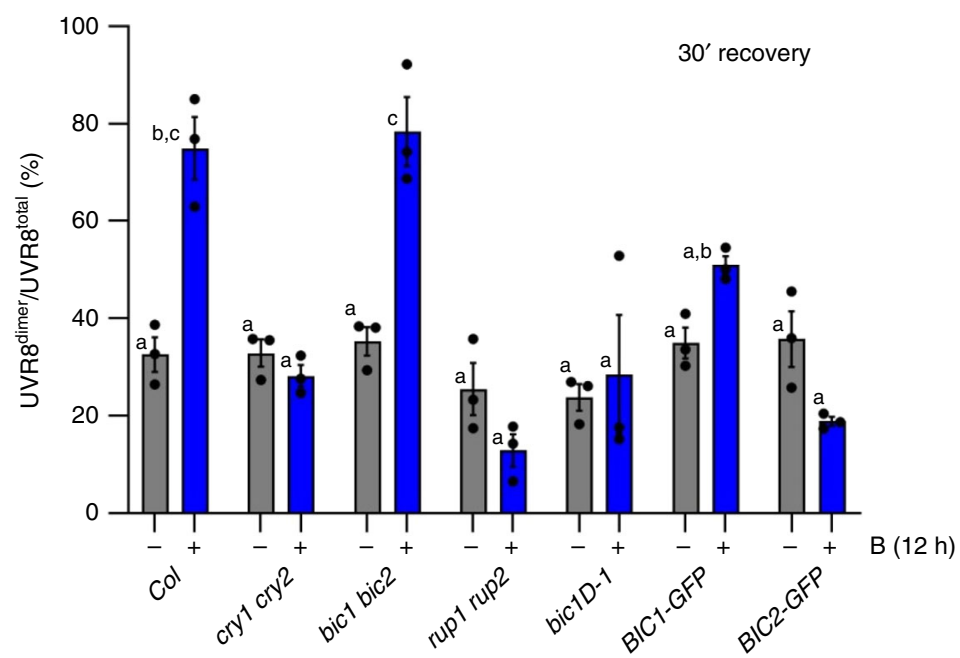

d
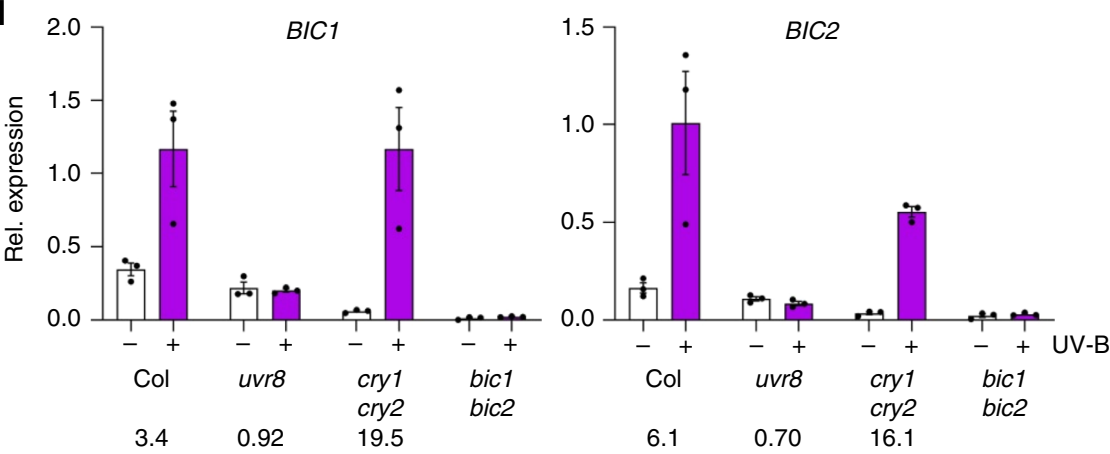

Fig. 3 BIC1 and BIC2 repress blue-light-induced RUP2 accumulation and UVR8 re-dimerization. a qRT-PCR analysis of RUP1 (left panel) and RUP2 (right panel) mRNA levels in 4-d-old Col, cry1 cry2, bic1 bic2, Pro 35 s:BIC1 (bic1D-1), Pro $35 s: B I C 1-G F P$ (BIC1-GFP), and Pro $35 s: B I C 2-G F P$ (BIC2-GFP) seedlings grown in darkness and exposed to blue light $\left(B ; 50 \mu \mathrm{mol} \mathrm{m}^{-2} \mathrm{~s}^{-1}\right)$ for $6 \mathrm{~h}(+)$ or not $(-)$. Data are means $\pm \mathrm{SEM}(N=3)$. $\mathbf{b}$ Immunoblot analysis of RUP2 protein level in 4-d-old Col, cry1 cry2, bic1 bic2, bic1-D1, BIC1-GFP, and BIC2-GFP seedlings grown in darkness and exposed to $12 \mathrm{~h}$ of blue light ( + ) or not (-). Actin is shown as protein loading control. c Quantification of the UVR8 dimer/UVR8 ${ }^{\text {total }}$ ratio (\%) after a 30-min recovery post-UV-B treatment in wild type (Col), cry1 cry2, bic1 bic2, rup1 rup2, bic1D-1, BIC1-GFP, and BIC2-GFP seedlings pre-treated with 12-h blue light $(+)$ or not $(-)$. Data are means \pm SEM $(N=3)$. Shared letters indicate no statistically significant difference in the means ( $P>0.05$; one-way ANOVA followed by post-hoc Tukey test). $\mathbf{d}$ qRT-PCR analysis of BIC1 (left panel) and BIC2 (right panel) mRNA levels in 4-d-old wild type (Col), uvr8-6 (uvr8), cry1-304 cry2-1 (cry1 cry2), and bic1-1 bic2-1 (bic1 bic2) seedlings grown in white light $\left(3.6 \mu \mathrm{mol} \mathrm{m} \mathrm{m}^{-2} \mathrm{~s}^{-1}\right)$ and exposed to UV-B for $3 \mathrm{~h}$ at $0.06 \mathrm{~mW} \mathrm{~cm}^{-2}(+)$ or not $(-)$. Data are means $\pm \mathrm{SEM}(\mathrm{N}=3)$; wild-type Col grown in white light without UV-B was set $=1$. Numbers below the graphs show the fold change values under +UV-B compared with that under $-U V-B$ for each genotype. 

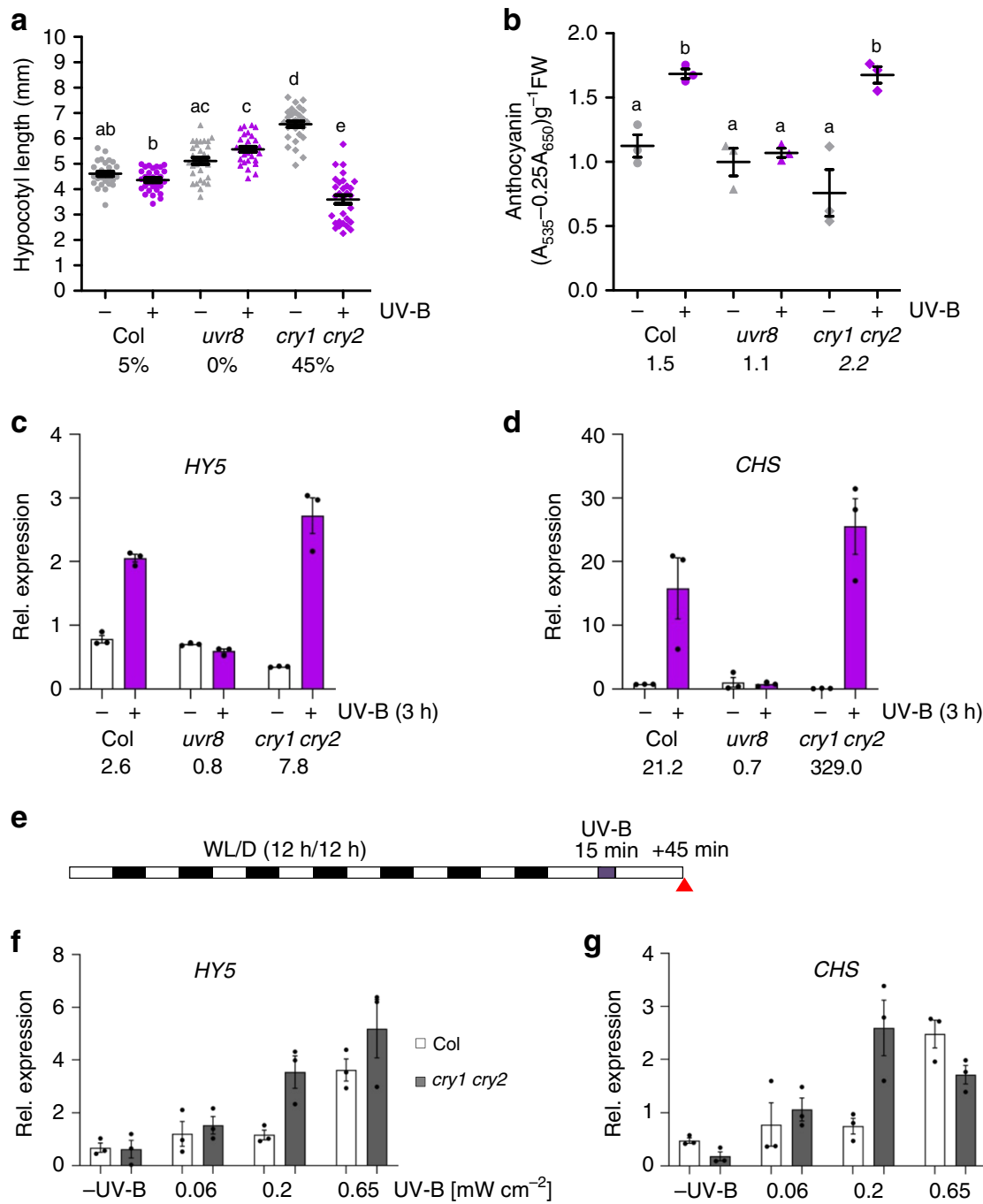

UV-B

$15 \mathrm{~min}+45 \mathrm{~min}$
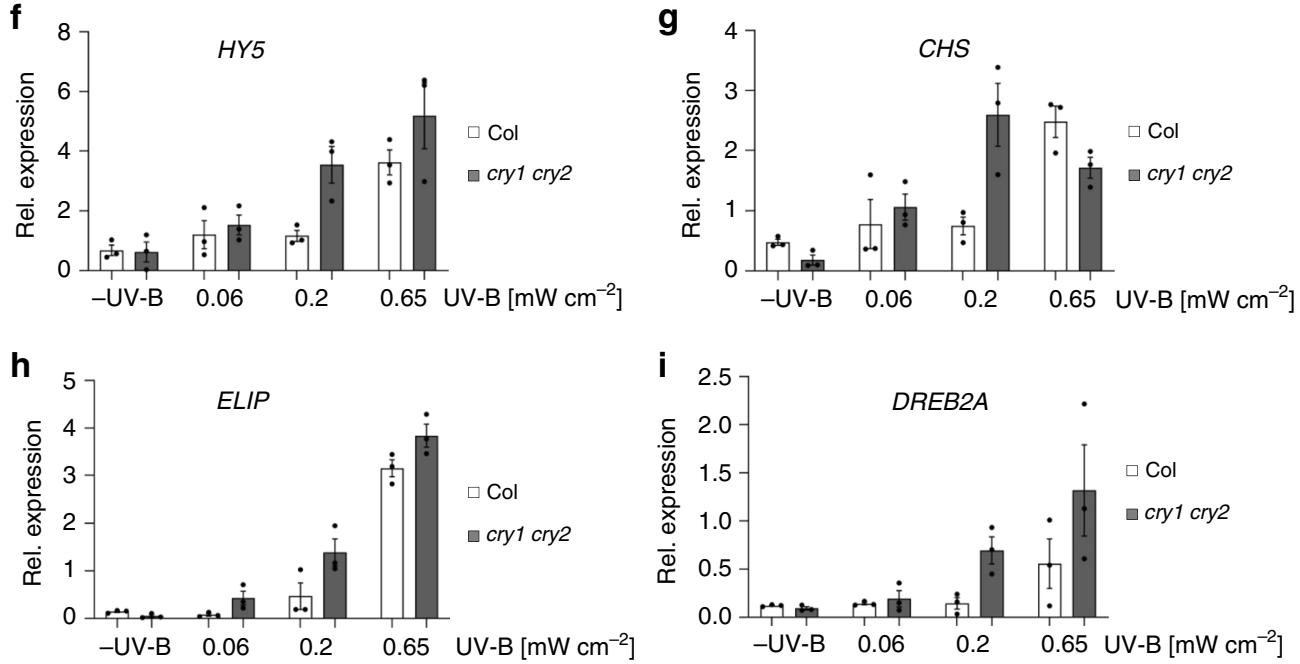

Fig. 4 cry1 and cry2 repress UVR8-mediated UV-B responses. a Quantification of hypocotyl length in 4-d-old wild type (Col), uvr8-6 (uvr8), and cry7-304 cry2-1 (cry1 cry2) seedlings grown in white light $\left(3.6 \mu \mathrm{mol} \mathrm{m}{ }^{-2} \mathrm{~s}^{-1}\right)$ supplemented with UV-B (+UV-B; $\left.0.015 \mathrm{~mW} \mathrm{~cm}^{-2}\right)$ or not $(-U V-B)$. Data are means \pm SD $(N=30)$. Shared letters indicate no statistically significant difference in the means $(P>0.05$; one-way ANOVA followed by post-hoc Tukey test). Numbers below bars show the relative hypocotyl growth inhibition by UV-B as a percentage. $\mathbf{b}$ Quantification of anthocyanin accumulation. Data are means \pm SEM $(N=3)$. Numbers below bars show the fold change values under +UV-B compared with that under $-U V-B$. $\mathbf{c}, \mathbf{d}$ qRT-PCR analysis of $\mathbf{c}$ HY5 and $\mathbf{d}$ CHS mRNA levels in 4-d-old Col, uvr8, and cry 1 cry2 seedlings grown in white light and exposed to UV-B at $0.06 \mathrm{~mW} \mathrm{~cm}^{-2}$ for $3 \mathrm{~h}(+)$ or not $(-)$. Data are means \pm SEM $(N=3)$; wild-type Col grown in white light without UV-B was set $=1$. Numbers below bars show the fold change values under $+U V-B$ compared with that under -UV-B for each genotype. e A schematic representation of the irradiation conditions. Seven-d-old seedling grown under 12-h light (WL)/12-h dark (D) cycles were irradiated for $15 \mathrm{~min}$ with broadband UV-B at different intensities $\left(0.06,0.20\right.$, and $\left.0.65 \mathrm{~mW} \mathrm{~cm}^{-2}\right)$ or not $(-U V)$. The red arrow indicates when plant samples were harvested 45 min following the UV-B treatment; see also ref. ${ }^{12}$. $\mathbf{f}-\mathbf{i}$ qRT-PCR analysis of $\mathbf{f} H Y 5$, $\mathbf{g}$ CHS, $\mathbf{h}$ ELIP2, and $\mathbf{i}$ DREB2A in Col and cry1 cry2 seedlings grown as per the schematic in panel (e). Data are means $\pm S E M(N=3)$.

2 (ELIP2), and DEHYDRATION-RESPONSIVE ELEMENT BINDING PROTEIN 2A (DREB2A) in a COP1- and UVR8dependent manner $5,12,29$. Although the response of the GFPcry1 overexpression line resembled wild type (Supplementary Fig. $3 c-f)$, the four tested genes showed consistently enhanced
UV-B-induced expression in cry1 cry 2 compared to wild type, in further agreement with UVR8 hyperactivity in cry1 cry2 (Fig. $4 \mathrm{f}-\mathrm{i}$ ). Our data from various distinct UV-B irradiation conditions indicate that cryptochrome signalling represses UVR8-mediated transcriptional responses. 


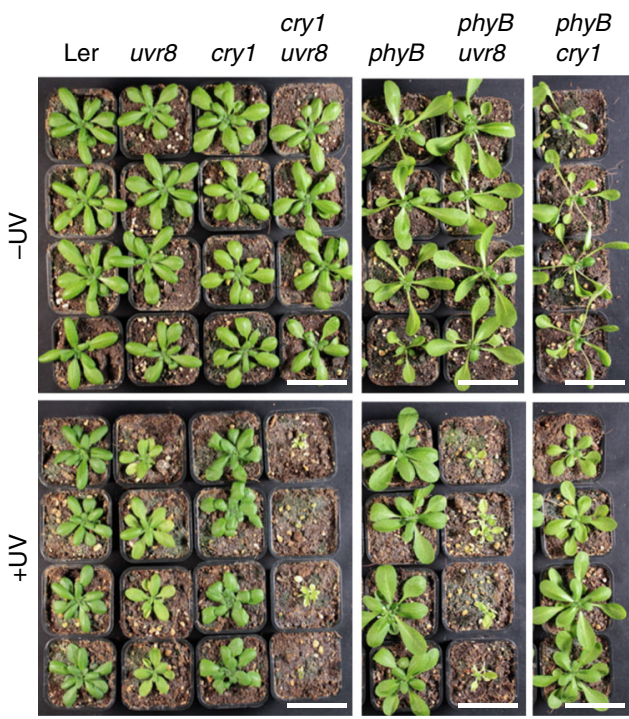

Fig. 5 Cryptochromes and phytochromes redundantly contribute to UVR8-mediated UV-B tolerance. Photographs of 28-d-old wild-type (Ler), uvr8-1 (uvr8), hy4-2.23N (cry1), hy4-2.23N uvr8-1 (cry1 uvr8), phyB-5 (phyB), phyB-5 uvr8-1 (phyB uvr8), and phyB-5 hy 4-2.23N (phyB cry1) plants grown under long-day conditions with $120 \mu \mathrm{mol} \mathrm{m} \mathrm{m}^{-2} \mathrm{~s}^{-1}$ of white light with supplemental UV-B (+UV, $\left.0.07 \mathrm{~mW} \mathrm{~cm}^{-2}\right)$ or not $(-U V)$. Bars $=5 \mathrm{~cm}$.

cryl and phyB establish UV-B tolerance alongside UVR8. Cryptochrome blue-light and phytochrome red-light signalling are known to induce expression of genes that largely overlap with UVR8-induced genes. These co-induced genes include those associated with UV-B tolerance, such as CHS and FLS; those encoding phenylpropanoid biosynthetic enzymes, which are important for the synthesis of flavonol glycosides that function as sunscreen metabolites; and PHR1 and UVR3, which encode photolyases important for DNA damage repair ${ }^{5,17,30}$. This indicates that, in addition to cryl negative regulation of UVR8 activity, cryl as well as phyB may contribute to UV-B tolerance alongside UVR8. In the absence of UV-B, the viability of cryl, $p h y B$, and $u v r 8$ single mutants and $c r y 1 u v r 8$, phyB uvr8, and phyB cry1 double mutants was not affected (Fig. 5). The main observable growth difference without UV-B was the long petioles of $p h y B$ plants that display a constitutive shade-response phenotype (Fig. 5), as described before ${ }^{31,32}$. In the presence of UV-B, cryl and wild-type plants showed comparable growth reductions (Fig. 5). In addition to the smaller rosette phenotype under UV-B, uvr8 plants displayed chlorotic leaves but remained viable (Fig. 5). Most strikingly, however, we observed that cryl uvr8 plants were extremely UV-B hypersensitive and did not survive under UV-B irradiation (Fig. 5). The constitutive shade-response phenotype of phyB evident without UV-B was clearly suppressed in the presence of UV-B (Fig. 5), in agreement with the finding that UVR8 antagonizes shade-avoidance responses ${ }^{33}$. phyB $u v r 8$ plant survival was also clearly impaired under UV-B, although $p h y B$ uvr 8 plants were less UV-B sensitive than cryl uvr8 (Fig. 5). Interestingly, in stark contrast to cry 1 uvr 8 and phyB uvr8 plants, cry 1 phyB double-mutant plants maintained UV-B tolerance (Fig. 5). We conclude that, in addition to the major role UVR8 plays in establishing UV-B acclimation and tolerance, blue light-activated cryl and red light-activated phyB contribute to full UV-B tolerance.

\section{Discussion}

Cellular signal transduction is usually composed of positive regulatory factors that function as "accelerators" as well as their specific negative feedback regulators that function as "brakes". Indeed, plant photoreceptors are activated upon photon absorption, which in conjunction with negative feedback regulation establishes a photoequilibrium according to the prevailing light conditions. For example, cryptochromes form an equilibrium between active homodimeric and inactive monomeric states, whereas UVR8 equilibrates between an active monomeric and an inactive homodimeric state ${ }^{6,17,34,35}$. Thus, both types of receptors are regulated at the level of their oligomeric state, with UVR8 "photomonomerizing" and cryptochromes "photodimerizing". Moreover, both cryptochromes and UVR8 activate expression of their own negative feedback regulators, namely BIC1/BIC2 and RUP1/RUP2, respectively ${ }^{13,17}$. These regulatory proteins interact directly with the respective photoreceptors and facilitate their reversion to the ground state and/or inhibit their activation ${ }^{13,14,17,18,34,36}$ (Fig. 6). Interestingly, BIC1 and BIC2 are strongly induced by UVR8 signalling, although BIC1 and BIC2 apparently do not directly function in UV-B responses. Reciprocally, RUP1 and RUP2 are strongly induced by cryptochrome signalling, although RUP1 and RUP2 do not function in responses to monochromatic blue light ${ }^{13}$. We show here that the UVR8 photoequilibrium is indeed responsive to cryptochromemediated blue-light signalling, thus identifying a novel photoreceptor co-action mechanism to balance UV-B sensitivity of plants under the polychromatic spectrum of sunlight (Fig. 6).

Phytochromes also mediate BIC1/BIC2 and RUP1/RUP2 transcriptional activation in response to red and far-red light ${ }^{13,18}$, suggesting that phytochromes contribute to the cross-regulation of cryptochromes and UVR8 through activation of their negative feedback regulators. However, the effect of phytochrome-mediated red light signalling on RUP1/RUP2 induction and UVR8 redimerization is very moderate if compared to the effect of blue light (Supplementary Fig. 5). In phyB signalling, positive feedback regulation is mediated by the phyB-interacting proteins PHOTOPERIODIC CONTROL OF HYPOCOTYL 1 (PCH1) and PCH1 LIKE (PCHL), which suppress phyB dark reversion from the active $\mathrm{Pfr}$ to the inactive $\mathrm{Pr}$ state ${ }^{37,38}$. Interestingly, in addition to red light, $P C H 1$ and $P C H L$ are transcriptionally activated by blue light and UV-B ${ }^{5,37}$. Indeed, blue light was shown to increase the response to red light facilitated by phy $\mathrm{B}^{37}$. Notwithstanding the physiological effects, the cross-regulation of these photoreceptor regulators is likely associated with COP1 inactivation and activation of HY5, which is the common signalling node of cryptochromes, phytochromes, and UVR 8 5, $, 10,20-23,34,39,40$. The convergence of these three wavelength-specific signalling pathways on COP1-HY5 is likely the basis for their overlapping photomorphogenic responses, which include hypocotyl growth inhibition, phenylpropanoid accumulation, flowering, and gene expression changes ${ }^{1-3,20,34,41}$. In particular, as demonstrated here, phytochrome, cryptochrome, and UVR8 signalling all contribute to establishing UV-B tolerance, suggesting that plants evolved photoreceptor cross-regulation mechanisms to globally balance their light responses in nature.

In contrast to the single mutants, cry 1 uvr 8 and $p h y B u v r 8$ double mutants were not viable under UV-B in our growth chamber experiments, demonstrating functional redundancies between cry1 and UVR8 as well as between phyB and UVR8. Interestingly, however, the cryl phyB double mutant could tolerate UV-B exposure, suggesting a major role for UVR8 even in the absence of both phyB and cryl. The potential contributions of the additional cryptochrome (cry2) and four phytochromes (phyA and phyC-E) remain to be determined. Moreover, phototropin 2 (phot2) plays a major role in chloroplast avoidance in response to high light ${ }^{42,43}$. As UV-B levels are high on sunny days and UV-B affects the photosynthetic machinery in chloroplasts $^{44,45}$, a contribution of phot2 to UV-B tolerance under natural conditions is to be anticipated. Independent of the 


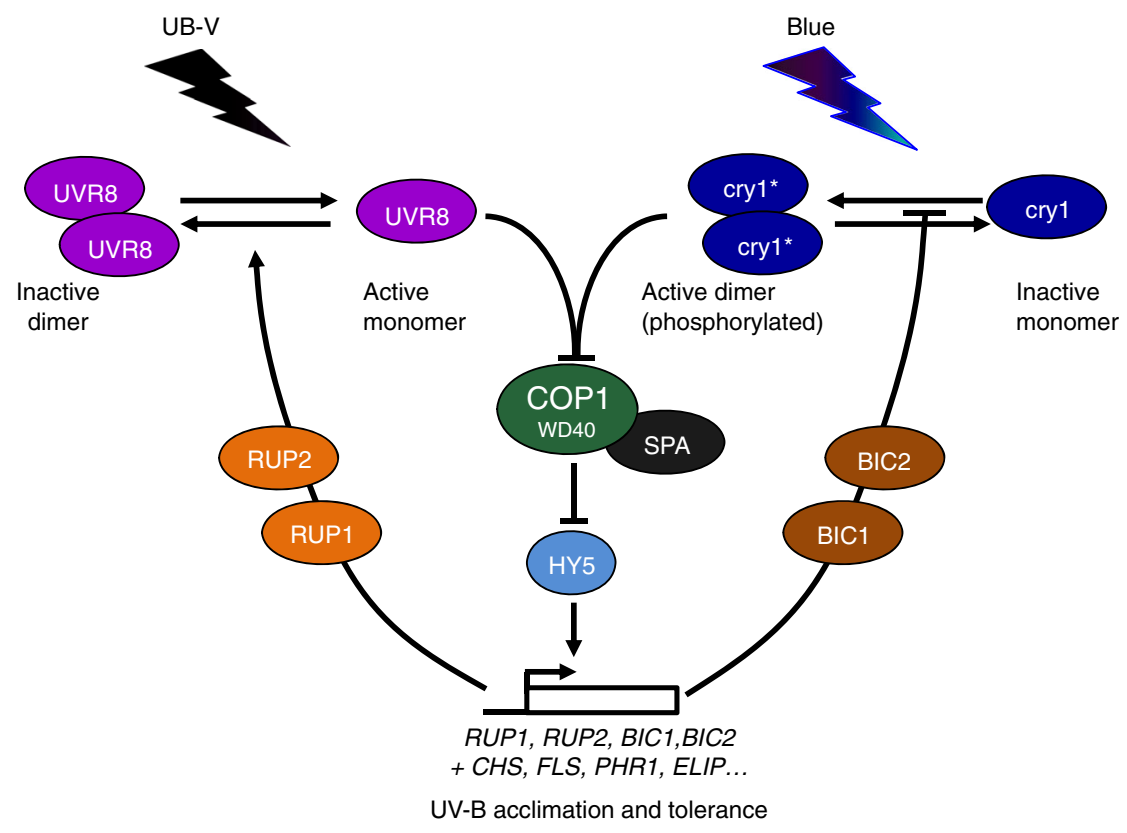

Fig. 6 Working model for the molecular integration of UVR8 and cryptochrome signalling. Light activation of UVR8 and cry1 leads to inhibition of the COP1-SPA E3 ubiquitin ligase complex, which releases HY5 from its repression through ubiquitination and proteasomal degradation. The transcriptional activator HY5 induces the expression of genes such as those associated with UV-B acclimation and tolerance (biosynthesis of sunscreen metabolites, DNA repair, photoprotection, etc.). HY5 also induces genes encoding distinct proteins with regulatory roles that concurrently provide feedback regulation of UVR8 and cryptochrome photoreceptor activities (note that the control of cry1 activity by UV-B through UVR8 signalling remains to be demonstrated). $\mathrm{BIC1}$ and BIC2 are thought to mainly block cry1 homodimerization, whereas RUP1 and RUP2 are thought to primarily facilitate UVR8 re-dimerization (although additionally blocking UVR8 monomerization cannot be excluded).

probable roles for additional photoreceptors in UV-B tolerance, recent work showed that triple-mutant plants lacking cry1, cry2, and UVR8 did not survive under natural sunlight, whereas they survived well when UV-B was specifically filtered out ${ }^{25}$. Thus, cryptochromes and UVR8 are indeed required for survival under natural conditions.

It is of note that our data provide evidence that the UVR8 signalling pathway is hyperactive in cry 1 cry 2 mutants and $\mathrm{BIC1} / \mathrm{BIC} 2$ overexpression lines. In contrast, the absence of enhanced RUP1 and RUP2 induction and associated lack of a clear effect on UVR8 activity in bic1 bic2 indicates that endogenous $\mathrm{BIC} 1$ and $\mathrm{BIC} 2$ do not play a major role in inhibiting cry activation under the conditions employed, including developmental stage, fluence rate, and kinetics. Notwithstanding this, the hyperactivity of UVR8 signalling in cry1 cry2 suggests that enhanced UVR8 signalling contributes to UV-B tolerance in the cryptochrome mutants. As red light also induces RUP1 and RUP2 expression, although to an apparently much lower level, a similar argument can be made for enhanced UVR8 signalling in phyB mutants. Moreover, as UVR8 signalling induces $B I C 1$ and $B I C 2$ expression, enhanced cryptochrome signalling in uvr8 single mutants may contribute to their UV-B tolerance; however, such a response remains to be shown. Thus, interactions within the network of phytochrome, cryptochrome, and UVR8 signalling pathways contribute to UV-B tolerance, allowing plant survival in sunlight in the natural environment. These cross-pathway interactions involve the commonly targeted COP1 E3 ubiquitin ligase and the upregulation of other photoreceptor-specific inhibitors. An exact understanding of these interactions and the individual contributions of the 13 Arabidopsis photoreceptors will provide a full appreciation of the roles photoreceptors play in plant survival. It is evident that RUP1 and RUP2 present a node for the integration of different light qualities allowing cross-regulation of the UVR8 activity state, which thus adapts UV-B tolerance strategies to the ambient light environment. It can also be speculated that other signalling pathways triggered by environmental signals beyond light perception may affect RUP1 and RUP2, thereby providing further cross-regulation of UVR8-mediated photomorphogenesis and UV-B acclimation.

\section{Methods}

Plant material, growth conditions, and UV-B irradiation. Arabidopsis thaliana mutants hy5-21546, uvr8-65, rup1-1, rup2-1, rup1-1 rup2-1 ${ }^{13}$, cry1-304, cry2-1, cry1304 cry2-1 ${ }^{47}$, phyA-211 ${ }^{48}$, cry1-304 cry2-1 phyA-412 ${ }^{49}$, phyB-9, phyA-211 phyB-937, bicl-1 bic2-1 $1^{17}$, as well as bic1D-1 $\left(\mathrm{Col} /\right.$ Pro $\left._{35}: B I C 1\right)$ and overexpression lines Pro $_{355}: B I C 1-G F P \# 18$, Pro $_{355}: B I C 2-G F P \# 47^{17}$, and $c r y 1-304 /$ Pro $_{355}: G F P-C R Y 1^{50}$ are all in the Columbia (Col) accession. Mutants uvr8-1 $1^{51}$, hy4-2.23N $N^{52}$, and phyB- $5^{53}$ are in the Landsberg erecta (Ler) accession. hy4-354, uvr8-75, and $u v r 8-7 /$ Pro $_{355}$ : YFP-UVR $8^{55}$ are in the Wassilewskija (Ws) accession.

Arabidopsis seeds were surface sterilized with sodium hypochlorite and sown on half-strength Murashige and Skoog basal salt medium (MS; Duchefa) containing $1 \%(\mathrm{w} / \mathrm{v})$ agar (Applichem) supplemented with $1 \%(\mathrm{w} / \mathrm{v})$ of sucrose, except for in those experiments involving etiolated seedlings. After 2-d stratification at $4^{\circ} \mathrm{C}$, seedlings were grown in white light or seeds were exposed to 6-h white light to induce germination before being grown in darkness at $22^{\circ} \mathrm{C}$.

For light-response experiments, light-emitting diode (LED) chambers (CLF floraLEDs, CLF Plant Climatics) were used (blue light: $50 \mu \mathrm{mol} \mathrm{m}^{-2} \mathrm{~s}^{-1}$, peak 470 $\mathrm{nm}$; red light: 50, 100 and $150 \mu \mathrm{mol} \mathrm{m} \mathrm{m}^{-2} \mathrm{~s}^{-1}$, peak $670 \mathrm{~nm}$; half-bandwidth of 20 $\mathrm{nm})$. White-light fluorescent tubes Osram L18W/30 $\left(3.6 \mu \mathrm{mol} \mathrm{m}^{-2} \mathrm{~s}^{-1}\right)$ were used in addition to supplemental UV-B from Philips TL20W/01RS narrowband UV-B tubes $\left(0.015\right.$ and $\left.0.06 \mathrm{~mW} \mathrm{~cm}^{-2}\right)$ or not ${ }^{29}$.

For UV-B tolerance assays, plants were grown on soil with a 16 -h day/8-h night cycle $\left(22^{\circ} \mathrm{C} / 18^{\circ} \mathrm{C}\right)$ in GroBanks (CLF Plant Climatics) with Philips Master TL-D $58 \mathrm{~W} / 840$ white-light fluorescent tubes $\left(120 \mu \mathrm{mol} \mathrm{m}^{-2} \mathrm{~s}^{-1}\right)$, supplemented or not with UV-B from Philips TL40W/01RS narrowband UV-B tubes $\left(0.07 \mathrm{~mW} \mathrm{~cm}^{-2}\right)^{41}$

Quantitative real-time PCR. Plant total RNA was isolated with the Plant RNeasy kit including DNase treatment according to the manufacturer's instructions (Qiagen). Synthesis of cDNA was performed using the TaqMan Reverse Transcription Reagents kit according to the manufacturer's standard protocol (Thermo Fisher Scientific). Each quantitative real-time PCR (qRT-PCR) reaction contained cDNA synthesized with a 1:1 mixture of oligo(dT) primers and random hexamers from $300 \mathrm{ng}$ of total RNA. qRT-PCR was performed using a PowerUp SYBR Green 
Master Mix (Thermo Fisher Scientific). PP2AA3 (PROTEIN PHOSPHATASE 2A SUBUNIT A3) was used as a reference gene ${ }^{56}$. The primers for RUP1, RUP1 qRT_fw (5'-AAG TGC CTG TTT CCG AGA GA-3') and RUP1_qRT_rv (5'-GTG GAT CCC ACA TTT GAA CC-3'), and for RUP2, RUP2_qRT_fw (5'-TTG TGG ATC GGA AAA CAA CA- $3^{\prime}$ ) and RUP2 qRT_rv (5'-CAC TGG TCC ACA CCT GAT TG-3') were used to quantify mRNA accumulation of RUP1 and RUP2, respectively. The others primers for qRT-PCR in this work were published as follows: BIC1 and $B I C 2^{17}, H Y 5$ and $C H S^{57}, D R E B 2 A^{58}$, and ELIP2 ${ }^{59}$.

Immunoblot analysis. For analysis of UVR8 dimers, proteins were extracted in $50 \mathrm{mM}$ Tris, $\mathrm{pH}$ 7.6, $150 \mathrm{mM} \mathrm{NaCl}, 2 \mathrm{mM}$ ethylenediaminetetraacetic acid (EDTA), 10\% (v/v) glycerol, $5 \mathrm{mM} \mathrm{MgCl} 2,1 \%$ (v/v) Igepal (Sigma), 1\% (v/v) protease inhibitor mixture for plant extracts (Sigma), and $10 \mu \mathrm{M}$ MG132. Twenty micrograms of proteins was separated by electrophoresis in $10 \%(\mathrm{wt} / \mathrm{v})$ SDS-polyacrylamide gels and gels were UV-B-irradiated before electrophoretic transfer to PVDF membrane ${ }^{6,14}$

For anti-RUP2 and anti-actin immunoblot analysis, total proteins were extracted in $50 \mathrm{mM}$ Na-phosphate (pH 7.4), $150 \mathrm{mM} \mathrm{NaCl}, 10 \%$ (v/v) glycerol, $5 \mathrm{mM}$ EDTA, $1 \mathrm{mM}$ dithiothreitol (DTT), 0.1\% (v/v) Triton X-100, $50 \mu \mathrm{M}$ MG132, $2 \mathrm{mM} \mathrm{Na}_{3} \mathrm{VO}_{4}, 2 \mathrm{mM} \mathrm{NaF}$, and $1 \%(\mathrm{v} / \mathrm{v})$ protease inhibitor mixture for plant extracts (P9599; Sigma-Aldrich). Protein concentration was determined using the Bio-Rad Protein Assay Dye Reagent Concentrate according to the manufacturer's instructions. Proteins were separated by $12 \%$ SDS-PAGE and transferred to nitrocellulose membranes. Anti-RUP2 ${ }^{(1-15)}(1: 500 \text { dilution })^{41}$, anti-UVR8 ${ }^{(426-440)}$ $(1: 4000)^{5}$, and anti-actin $(1: 20,000)$ (A0480, Sigma-Aldrich) were used as the primary antibodies. Horseradish peroxidase (HRP)-conjugated anti-rabbit and anti-mouse immunoglobulins (Dako A/S) were used as the secondary antibodies $(1: 20,000)$. Immunodetection was performed using an ECL Plus Western Detection Kit and revealed with an ImageQuant LAS 4000 mini CCD camera system (GE Healthcare). For quantitative analysis of UVR8 re-dimerization, immunoblot membranes were probed with IRDye 800 fluorescent dye-coupled anti-rabbit secondary antibodies $(1: 10,000)$ and analysed using the Odyssey ${ }^{\circledR}$ IR imaging system (LI-COR Biosciences). ImageJ was used for quantifications, for which bands intensities for UVR8 dimers and UVR8 monomers were added to represent $100 \%$. Data are presented as percentage of UVR $8^{\text {dimer }}$ amounts (UVR $8^{\text {dimer }} / \mathrm{UVR} 8^{\text {total }}$ ).

Hypocotyl length measurements. Four-day-old Arabidopsis seedlings were grown under continuous irradiation in a light field with Osram L18W/30 tubes $\left(3.6 \mu \mathrm{mol} \mathrm{m}{ }^{-2} \mathrm{~s}^{-1}\right.$; measured with a LI-250 Light Meter; LI-COR Biosciences) supplemented with Philips TL20W/01RS narrowband UV-B tubes. The UV-B range was modulated by the use of 3-mm transmission cut-off filters of the WG series with half-maximal transmission at $305 \mathrm{~nm}$ (WG305, +UV-B at $0.06 \mathrm{~mW} \mathrm{~cm}^{-2}$; measured with a VLX-3W UV Light Meter equipped with a CX-312 sensor; Vilber Lourmat), at $320 \mathrm{~nm}$ (WG320, +UV-B at $0.015 \mathrm{~mW} \mathrm{~cm}^{-2}$ ), or $345 \mathrm{~nm}$ (WG345, -UV-B) (Schott Glaswerke) ${ }^{5,29}$. Seedlings were scanned and their hypocotyl lengths were measured $(N=30)$ using ImageJ software. Experiments were independently repeated three times.

Anthocyanin extraction and measurement. Plant seedlings were grown $4 \mathrm{~d}$ in the same conditions as for hypocotyl length measurement, and $50 \mathrm{mg}$ plant material was homogenized for $10 \mathrm{~s}$ using a Silamat S5 mixer (Ivoclar Vivadent). $250 \mu \mathrm{l}$ of acidic methanol $(1 \% \mathrm{HCl})$ was added to each sample and placed in an overhead shaker at $4^{\circ} \mathrm{C}$ for $1 \mathrm{~h}$. Samples were centrifuged for $1 \mathrm{~min}$ at $14,000 \mathrm{rpm}$ and the supernatant was used to quantify anthocyanin content based on the absorption at 535 and $650 \mathrm{~nm}$. Values were reported as $\mathrm{A}_{535}-0.25\left(\mathrm{~A}_{650}\right) \mathrm{g}^{-1}$ fresh weight.

Statistical analyses. Statistical analyses were performed using GraphPad Software Prism 8 software (San Diego, California). Statistical significance of the differences between means was determined using Student's $t$ tests (protein quantification data) or one-way ANOVA and post-hoc Tukey tests (hypocotyl length data).

\section{Data availability}

The data supporting the findings in this study are available and described within the paper and its supplementary files. The source data underlying Figs. 1-5 and Supplementary Figs. $1-5$ are provided as a separate Source Data file. All biological material generated in this study are available from the corresponding author upon reasonable request.

Received: 4 October 2019; Accepted: 18 February 2020;

Published online: 12 March 2020

\section{References}

1. Galvao, V. C. \& Fankhauser, C. Sensing the light environment in plants: photoreceptors and early signaling steps. Curr. Opin. Neurobiol. 34, 46-53 (2015).
2. Jenkins, G. I. Photomorphogenic responses to ultraviolet-B light. Plant Cell Environ. 40, 2544-2557 (2017).

3. Yin, R. \& Ulm, R. How plants cope with UV-B: from perception to response Curr. Opin. Plant Biol. 37, 42-48 (2017).

4. Liang, T., Yang, Y. \& Liu, H. Signal transduction mediated by the plant UV-B photoreceptor UVR8. New Phytol. 221, 1247-1252 (2019).

5. Favory, J. J. et al. Interaction of COP1 and UVR8 regulates UV-B-induced photomorphogenesis and stress acclimation in Arabidopsis. EMBO J. 28, 591-601 (2009).

6. Rizzini, L. et al. Perception of UV-B by the Arabidopsis UVR8. Science 332, 103-106 (2011).

7. Tilbrook, K. et al. UV-B perception and acclimation in Chlamydomonas reinhardtii. Plant Cell 28, 966-983 (2016).

8. Yin, R., Arongaus, A. B., Binkert, M. \& Ulm, R. Two distinct domains of the UVR8 photoreceptor interact with COP1 to initiate UV-B signaling in Arabidopsis. Plant Cell 27, 202-213 (2015).

9. Lau, K., Podolec, R., Chappuis, R., Ulm, R. \& Hothorn, M. Plant photoreceptors and their signaling components compete for COP1 binding via VP peptide motifs. EMBO J. 38, e102140 (2019).

10. Binkert, M. et al. UV-B-responsive association of the Arabidopsis bZIP transcription factor ELONGATED HYPOCOTYL5 with target genes, including its own promoter. Plant Cell 26, 4200-4213 (2014).

11. Huang, X. et al. Arabidopsis FHY3 and HY5 positively mediate induction of COP1 transcription in response to photomorphogenic UV-B light. Plant Cell 24, 4590-4606 (2012).

12. Ulm, R. et al. Genome-wide analysis of gene expression reveals function of the bZIP transcription factor HY5 in the UV-B response of Arabidopsis. Proc. Natl Acad. Sci. USA 101, 1397-1402 (2004).

13. Gruber, H. et al. Negative feedback regulation of UV-B-induced photomorphogenesis and stress acclimation in Arabidopsis. Proc. Natl Acad. Sci. USA 107, 20132-20137 (2010).

14. Heijde, M. \& Ulm, R. Reversion of the Arabidopsis UV-B photoreceptor UVR8 to the homodimeric ground state. Proc. Natl Acad. Sci. USA 110, 1113-1118 (2013)

15. Findlay, K. M. \& Jenkins, G. I. Regulation of UVR8 photoreceptor dimer/ monomer photo-equilibrium in Arabidopsis plants grown under photoperiodic conditions. Plant Cell Environ. 39, 1706-1714 (2016).

16. Ren, H. et al. Two E3 ligases antagonistically regulate the UV-B response in Arabidopsis. Proc. Natl Acad. Sci. USA 116, 4722-4731 (2019).

17. Wang, Q. et al. Photoactivation and inactivation of Arabidopsis cryptochrome 2. Science 354, 343-347 (2016).

18. Wang, X. et al. A CRY-BIC negative-feedback circuitry regulating blue light sensitivity of Arabidopsis. Plant J. 92, 426-436 (2017).

19. Hoecker, U. The activities of the E3 ubiquitin ligase COP1/SPA, a key repressor in light signaling. Curr. Opin. Plant Biol. 37, 63-69 (2017)

20. Podolec, R. \& Ulm, R. Photoreceptor-mediated regulation of the COP1/SPA E3 ubiquitin ligase. Curr. Opin. Plant Biol. 45, 18-25 (2018).

21. Lian, H. L. et al. Blue-light-dependent interaction of cryptochrome 1 with SPA1 defines a dynamic signaling mechanism. Genes Dev. 25, 1023-1028 (2011).

22. Liu, B., Zuo, Z., Liu, H., Liu, X. \& Lin, C. Arabidopsis cryptochrome 1 interacts with SPA1 to suppress COP1 activity in response to blue light. Genes Dev. 25, 1029-1034 (2011).

23. Zuo, Z., Liu, H., Liu, B., Liu, X. \& Lin, C. Blue light-dependent interaction of CRY2 with SPA1 regulates COP1 activity and floral initiation in Arabidopsis. Curr. Biol. 21, 841-847 (2011).

24. Holtkotte, X., Ponnu, J., Ahmad, M. \& Hoecker, U. The blue light-induced interaction of cryptochrome 1 with COP1 requires SPA proteins during Arabidopsis light signaling. PLoS Genet. 13, e1007044 (2017).

25. Rai, N. et al. How do cryptochromes and UVR8 interact in natural and simulated sunlight? J. Exp. Bot. 70, 4975-4990 (2019).

26. Wade, H. K., Bibikova, T. N., Valentine, W. J. \& Jenkins, G. I. Interactions within a network of phytochrome, cryptochrome and UV-B phototransduction pathways regulate chalcone synthase gene expression in Arabidopsis leaf tissue. Plant J. 25, 675-685 (2001).

27. Morales, L. O. et al. Multiple roles for UV RESISTANCE LOCUS8 in regulating gene expression and metabolite accumulation in Arabidopsis under solar ultraviolet radiation. Plant Physiol. 161, 744-759 (2013).

28. Fuglevand, G., Jackson, J. A. \& Jenkins, G. I. UV-B, UV-A, and blue light signal transduction pathways interact synergistically to regulate chalcone synthase gene expression in Arabidopsis. Plant Cell 8, 2347-2357 (1996).

29. Oravecz, A. et al. CONSTITUTIVELY PHOTOMORPHOGENIC1 is required for the UV-B response in Arabidopsis. Plant Cell 18, 1975-1990 (2006).

30. Tepperman, J. M. et al. Expression profiling of phyB mutant demonstrates substantial contribution of other phytochromes to red-light-regulated gene expression during seedling de-etiolation. Plant J. 38, 725-739 (2004).

31. Franklin, K. A. et al. Phytochromes B, D, and E act redundantly to control multiple physiological responses in Arabidopsis. Plant Physiol. 131, 1340-1346 (2003) 
32. Lorrain, S., Allen, T., Duek, P. D., Whitelam, G. C. \& Fankhauser, C. Phytochrome-mediated inhibition of shade avoidance involves degradation of growth-promoting bHLH transcription factors. Plant J. 53, 312-323 (2008).

33. Hayes, S., Velanis, C. N., Jenkins, G. I. \& Franklin, K. A. UV-B detected by the UVR8 photoreceptor antagonizes auxin signaling and plant shade avoidance. Proc. Natl Acad. Sci. USA 111, 11894-11899 (2014).

34. Wang, Q. et al. Beyond the photocycle-how cryptochromes regulate photoresponses in plants? Curr. Opin. Plant Biol. 45, 120-126 (2018).

35. Heijde, M. \& Ulm, R. UV-B photoreceptor-mediated signalling in plants. Trends Plant Sci. 17, 230-237 (2012).

36. Tilbrook, K. et al. The UVR8 UV-B photoreceptor: perception, signaling and response. Arabidopsis Book 11, e0164 (2013).

37. Enderle, B. et al. PCH1 and PCHL promote photomorphogenesis in plants by controlling phytochrome B dark reversion. Nat. Commun. 8, 2221 (2017).

38. Huang, $\mathrm{H}$. et al. $\mathrm{PCH} 1$ regulates light, temperature, and circadian signaling as a structural component of phytochrome B-photobodies in Arabidopsis. Proc. Natl Acad. Sci. USA 116, 8603-8608 (2019).

39. Lu, X. D. et al. Red-light-dependent interaction of phyB with SPA1 promotes COP1-SPA1 dissociation and photomorphogenic development in Arabidopsis. Mol. Plant 8, 467-478 (2015).

40. Sheerin, D. J. et al. Light-activated phytochrome A and B interact with members of the SPA family to promote photomorphogenesis in Arabidopsis by reorganizing the COP1/SPA complex. Plant Cell 27, 189-201 (2015).

41. Arongaus, A. B. et al. Arabidopsis RUP2 represses UVR8-mediated flowering in noninductive photoperiods. Genes Dev. 32, 1332-1343 (2018).

42. Kasahara, M. et al. Chloroplast avoidance movement reduces photodamage in plants. Nature 420, 829-832 (2002).

43. Cazzaniga, S., Dall' Osto, L., Kong, S. G., Wada, M. \& Bassi, R. Interaction between avoidance of photon absorption, excess energy dissipation and zeaxanthin synthesis against photooxidative stress in Arabidopsis. Plant J. 76, 568-579 (2013).

44. Demarsy, E., Goldschmidt-Clermont, M. \& Ulm, R. Coping with 'dark sides of the sun' through photoreceptor signaling. Trends Plant Sci. 23, 260-271 (2018).

45. Takahashi, S. et al. The solar action spectrum of photosystem II damage. Plant Physiol. 153, 988-993 (2010).

46. Oyama, T., Shimura, Y. \& Okada, K. The Arabidopsis HY5 gene encodes a bZIP protein that regulates stimulus-induced development of root and hypocotyl. Genes Dev. 11, 2983-2995 (1997).

47. Mockler, T. C., Guo, H., Yang, H., Duong, H. \& Lin, C. Antagonistic actions of Arabidopsis cryptochromes and phytochrome B in the regulation of floral induction. Development 126, 2073-2082 (1999).

48. Nagatani, A., Reed, J. W. \& Chory, J. Isolation and initial characterization of Arabidopsis mutants that are deficient in phytochrome A. Plant Physiol. 102, 269-277 (1993).

49. Mockler, T. et al. Regulation of photoperiodic flowering by Arabidopsis photoreceptors. Proc. Natl Acad. Sci. USA 100, 2140-2145 (2003).

50. Gao, J. et al. Trp triad-dependent rapid photoreduction is not required for the function of Arabidopsis CRY1. Proc. Natl Acad. Sci. USA 112, 9135-9140 (2015).

51. Kliebenstein, D. J., Lim, J. E., Landry, L. G. \& Last, R. L. Arabidopsis UVR8 regulates ultraviolet- $\mathrm{B}$ signal transduction and tolerance and contains sequence similarity to human regulator of chromatin condensation 1. Plant Physiol. 130, 234-243 (2002).

52. Koornneef, M., Rolff, E. \& Spruit, C. J. P. Genetic control of light-inhibited hypocotyl elongation in Arabidopsis thaliana (L.) Heynh. Z. Pflanzenphysiol. 100, 147-160 (1980).

53. Reed, J. W., Nagatani, A., Elich, T. D., Fagan, M. \& Chory, J. Phytochrome A and phytochrome B have overlapping but distinct functions in Arabidopsis development. Plant Physiol. 104, 1139-1149 (1994).

54. Ahmad, M. \& Cashmore, A. R. HY4 gene of $A$. thaliana encodes a protein with characteristics of a blue-light photoreceptor. Nature 366, 162-166 (1993).
55. Yin, R., Skvortsova, M. Y., Loubery, S. \& Ulm, R. COP1 is required for UV-Binduced nuclear accumulation of the UVR8 photoreceptor. Proc. Natl Acad. Sci. USA 113, E4415-E4422 (2016).

56. Czechowski, T., Stitt, M., Altmann, T., Udvardi, M. K. \& Scheible, W. R. Genome-wide identification and testing of superior reference genes for transcript normalization in Arabidopsis. Plant Physiol. 139, 5-17 (2005).

57. $\mathrm{Xu}, \mathrm{D}$. et al. BBX21, an Arabidopsis B-box protein, directly activates HY5 and is targeted by COP1 for $26 \mathrm{~S}$ proteasome-mediated degradation. Proc. Natl Acad. Sci. USA 113, 7655-7660 (2016).

58. Morimoto, K. et al. BPM-CUL3 E3 ligase modulates thermotolerance by facilitating negative regulatory domain-mediated degradation of DREB2A in Arabidopsis. Proc. Natl Acad. Sci. USA 114, E8528-E8536 (2017).

59. Hayami, N. et al. The responses of Arabidopsis Early Light-Induced Protein 2 to ultraviolet $\mathrm{B}$, high light, and cold stress are regulated by a transcriptional regulatory unit composed of two elements. Plant Physiol. 169, 840-855 (2015).

\section{Acknowledgements}

We would like to thank Emilie Demarsy and Michel Goldschmidt-Clermont for helpful comments on the manuscript, and Xu Wang and Chentao Lin for providing the bic1 bic2 double mutant as well as BIC1, BIC2 and CRY1 overexpression lines. This work was supported by the University of Geneva, the Ernst and Lucie Schmidheiny Foundation, and the Swiss National Science Foundation (grants nos. 31003A_175774 and IZSAZ3_173361).

\section{Author contributions}

N.T. and R.U. designed the study; N.T. performed the experiments; N.T. and R.U. analysed the data; and N.T. and R.U. wrote the paper.

\section{Competing interests}

The authors declare no competing interests.

\section{Additional information}

Supplementary information is available for this paper at https://doi.org/10.1038/s41467020-15133-y.

Correspondence and requests for materials should be addressed to R.U.

Peer review information Nature Communications thanks Hongtao Liu and the other, anonymous, reviewer(s) for their contribution to the peer review of this work.

Reprints and permission information is available at http://www.nature.com/reprints

Publisher's note Springer Nature remains neutral with regard to jurisdictional claims in published maps and institutional affiliations.

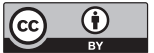

Open Access This article is licensed under a Creative Commons Attribution 4.0 International License, which permits use, sharing, adaptation, distribution and reproduction in any medium or format, as long as you give appropriate credit to the original author(s) and the source, provide a link to the Creative Commons license, and indicate if changes were made. The images or other third party material in this article are included in the article's Creative Commons license, unless indicated otherwise in a credit line to the material. If material is not included in the article's Creative Commons license and your intended use is not permitted by statutory regulation or exceeds the permitted use, you will need to obtain permission directly from the copyright holder. To view a copy of this license, visit http://creativecommons.org/ licenses/by/4.0/.

(c) The Author(s) 2020 